\title{
Hepatic vein thrombosis associated with segmental hypo-attenuation in the liver: an unusual complication of a haemodialysis catheter
}

\author{
Alexandra Lapeyre-Prost • Olivier Clément • \\ Emad Lotfalizadeh • Véronique Boussaud • \\ Jean-Luc Diehl
}

Received: 13 December 2014/ Accepted: 20 December 2014/Published online: 9 January 2015 (C) SIMI 2015

One of the main complications of hemodialysis catheters is upper extremity deep venous thrombosis. Although femoral, subclavian, jugular, axillary or superior vena cava thrombosis are well-known complications, hepatic vein thrombosis resulting from accidental hepatic venous catheterization has been reported only once [1].

A 31-year-old woman was referred because of an acute abdominal pain. Physical examination revealed tenderness and guarding on palpation of the epigastric region. A laboratory examination revealed anicteric cholestasis and severe hyperkalemia.

The medical history was marked by bi-pulmonary transplantation for cystic fibrosis, further complicated by diabetes and vascular nephropathy with the need of chronic hemodialysis. A long-term dialysis catheter had been inserted via the right jugular vein 2 months ago.

An emergency dialysis was performed to control severe hyperkalemia. A CT scan revealed on portal venous phase, a well-marginated heterogeneously enhanced liver, with hypodensity of the left lobe, and a thrombosis of medium and left hepatic veins, in association with a loop of the

A. Lapeyre-Prost · J.-L. Diehl ( $₫)$

Medical Intensive Care Unit and Inserm UMR-S1140, Georges Pompidou European Hospital, Assistance Publique-Hopitaux de Paris, Paris Descartes University, Paris, France

e-mail: jldiehl@invivo.edu

O. Clément · E. Lotfalizadeh

Department of Radiology, Georges Pompidou European Hospital, Assistance Publique-Hopitaux de Paris, Paris Descartes University, Paris, France

V. Boussaud

Department of Cardiovascular Surgery, Georges Pompidou European Hospital, Assistance Publique-Hopitaux de Paris, Paris Descartes University, Paris, France dialysis catheter at the intersection of these two veins (Fig. 1). Unfractionated heparin therapy was immediately started, with subsequent introduction of Vitamin $\mathrm{K}$ antagonist (VKA) for 3 months. The long-term dialysis catheter was removed, followed by the insertion of a new one. The clinical symptoms fully resolved within $72 \mathrm{~h}$. A complete resolution of the thrombosis was evidenced on an echoDoppler performed after 3 months of VKA therapy.

Usually, the tip of a jugular venous catheter should be located at the junction of the right atrium and of the superior vena cava. Initial mal-positioning of a catheter increases the risk of catheter-related deep venous thrombosis [2]. In the present case, positioning of the catheter was not rigorously assessed, leading to a delayed complication of hepatic vein thrombosis.

A characteristic CT pattern of parenchymal perfusion following bolus intravenous injection of contrast material is usually described in the Budd-Chiari syndrome. It consists of early homogenous central enhancement together with delayed patchy enhancement of the periphery of the liver, and prolonged retention of the contrast medium in the periphery [3]. In a few cases, hypodensity of well-marginated areas of the liver is reported in the context of hepatic vein obstruction, probably due to portal flow inversion, the vascularisation of the occluded area being also supplied with arterial blood alone [4, 5].

We found in our case, a similar pattern of low attenuation of the left liver lobe in association with a left hepatic vein occlusion. To the best of our knowledge, only one case of segmental attenuation of the liver has been reported in a patient with right hepatic vein thrombosis related to central venous catheterization [1].

The present case stresses the importance of rigorously checking the positioning of the long-term dialysis catheters. It illustrates the possibility of delayed thrombotic 
Fig. 1 Mal-positioning of the catheter at the intersection of medium and left hepatic vein as evidenced on contrast enhanced CT scan (a) and venous hepatic thrombosis (thick arrow) near to the tip of the catheter (double arrow) on portal venous phase of enhanced axial CT scan (b). A hypo-attenuation of the left liver lobe with sharp delimitation (thin arrows) was also observed on enhanced axial CT scan (b)
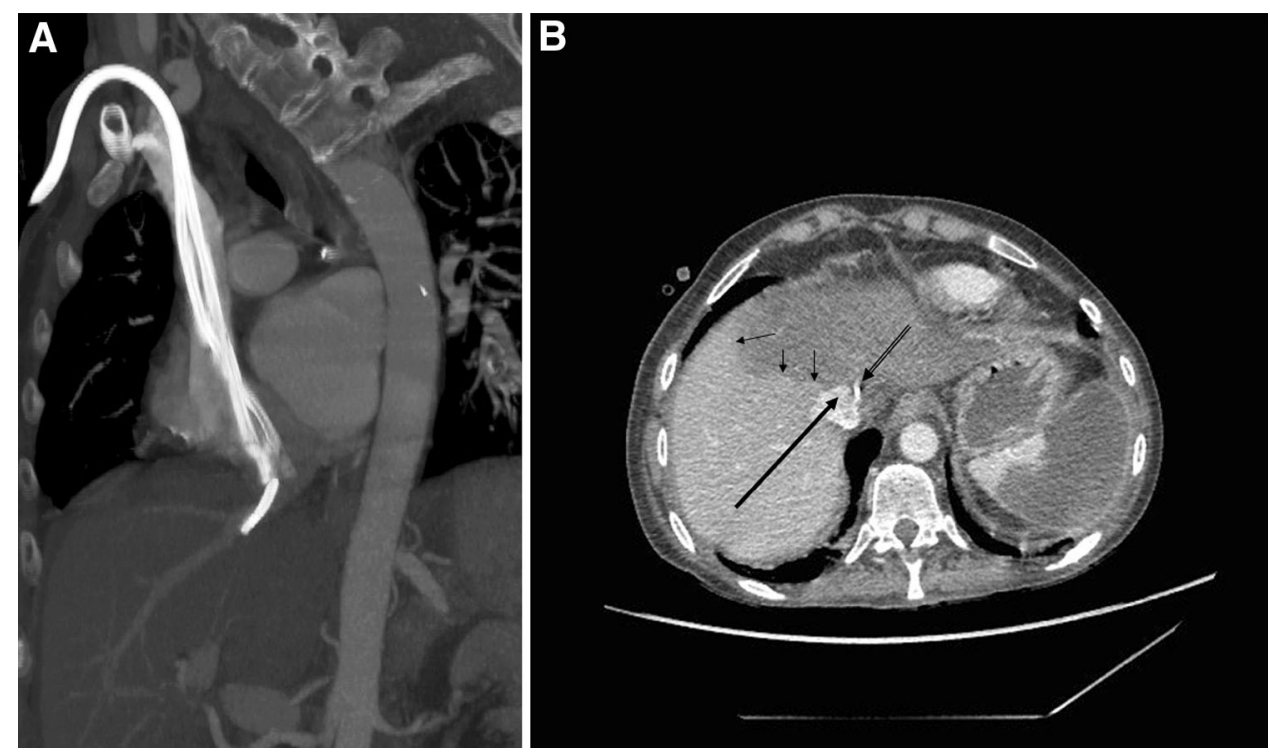

consequences in case of positioning of the catheter in the hepatic veins. Moreover, we report for the first time a CT aspect of focal hypo-attenuation in the liver in association with a long-term dialysis catheter-related hepatic vein thrombosis. However, at this time, such an aspect could only be suggestive of hepatic vein thrombosis, which has to be proved by direct observation of the thrombus.

\section{Conflict of interest None.}

\section{References}

1. Fitoz S, Atasoy Ç, Yagmurlu A, Akyar S (2002) Segmental hyperattenuation in the liver as a result of right hepatic vein thrombosis: an unusual complication of central venous catheterization. Australas Radiol 46:299-301

2. Gibson F, Bodenham A (2013) Misplaced central venous catheters: applied anatomy and practical management. $\mathrm{Br} \mathrm{J}$ Anaesth 110:333-346

3. Gryspeerdt S, Van Hoe L, Marchal G, Baert AL (1997) Evaluation of hepatic perfusion disorders with double-phase spiral CT. Radiographics 17:337-348

4. Murata S, Itai $Y$, Asato $M$ et al (1995) Effect of temporary occlusion of the hepatic vein on dual blood in the liver: evaluation with spiral CT. Radiology 197:351-356

5. Lautt WW (1985) Mechanism and role of intrinsic regulation of hepatic arterial blood flow: hepatic arterial buffer response. Am J Physiol 1985(249):G549-G556 